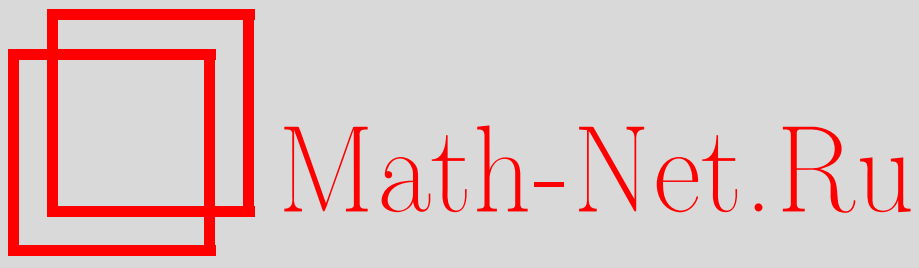

С. В. Матвеев, Обобщенные граф-многообразия и их эффективное распознавание, Матем. сб., 1998, том 189, номер 10, 89-104

DOI: https://doi.org/10.4213/sm352

Использование Общероссийского математического портала Math-Net.Ru подразумевает, что вы прочитали и согласны с пользовательским соглашением http://www . mathnet.ru/rus/agreement

Параметры загрузки:

IP : 35.173 .219 .12

26 апреля 2023 г., $17: 37: 47$ 
УДК 513.83

\author{
C.В. Матвеев
}

\title{
Обобщенные граф-многообразия и их эффективное распознавание
}

\begin{abstract}
Под обобщенным граф-многообразием понимается трехмерное многообразие, получающееся склеиванием элементарных блоков, где каждый элементарный блок либо является многообразием Зейферта, либо не содержит существенных торов и колец. Из известной теоремы о торическом разбиении следует, что любое компактное трехмерное многообразие, край которого либо пуст, либо состоит из торов, имеет каноническое представление в виде обобщенного граф-многообразия. В статье приводится простое и короткое независимое доказательство существования канонического представления и описывается частичный алгоритм его построения. Приводится также простой критерий гиперболичности блоков, не являющихся многоообразиями Зейферта.

Библиография: 10 названий.
\end{abstract}

\section{§1. Введение}

Пусть $M$ - компактное ориентируемое неприводимое трехмерное многообразие, край которого либо пуст, либо состоит из несжимаемых торов. Тогда из знаменитой теоремы о разбиении торами [1], [2] следует, что многообразие $M$ содержит конечную каноническую систему торов и колец, которая разбивает его на многообразия Зейферта и многообразия, уже не содержащие существенных торов и колец. Взаимное расположение этих частей удобно описывать с помощью некоторого графа, аналогично тому, как это делается при изучении графф-многообразий Вальдхаузена [3] или при построении топологических инвариантов интегрируемых гамильтоновых систем [4].

В этом смысле любое трехмерное многообразие является обобщенным графф-многообразием. Оригинальные доказательства этого результата в [1] и [2] довольно сложны, поскольку попутно раскрывают ряд важных свойств характеристических подмногообразий. Предлагаемое здесь прямое доказательство намного проше и короче.

Проблема отыскания канонической системы разрезающих торов имеет алгоритмическое решение. Однако оно основано на теории нормальных поверхностей В. Хакена и поэтому носит чисто теоретический характер. Мы предлагаем частичный практический алгоритм, который основан на теории специальных спайнов (см. [5]) и может быть реализован на компютере (см. [6]). Алгоритм разбивает данное многообразие на многообразия Зейферта и на части, которые он не может распознать и которые часто оказываются гиперболическими. Мы описываем также простой способ (основанный на одном наблюдении А. Кассона), которьй в 
некоторых случаях позволяет доказать, что данная неопознанная часть не содержит несжимаемых торов и колец и поэтому обладает гиперболической структурой. В случае успешной работы обоих алгоритмов мы получаем явное описание многообразия $M$ в виде суммы зейфертовых и гиперболических частей вместе с информацией о том, как эти части склеены между собой. Поскольку такое разбиение единственно, эти данные можно рассматривать как “имя" многообразия, которое отличает его от всех других многообразий. Это сводит проблему распознавания для многообразий к сравнению их имен.

Автор благодарит Российский фонд фундаментальных исследований за финансовую поддержку данной работы (грант № 96-01-00847), а также институт Макса Планка в Бонне, где был написан окончательньй вариант статьи.

\section{§ 2. Обобщенные граф-многообразия}

Напомним некоторые основные понятия трехмерной топологии. Трехмерное многообразие $M$ называется неприводимым, если каждая 2-сфера $S \subset M$ ограничивает в $M$ трехмерный шар. Поверхность $F \subset M$ сжимаема, если найдется такой диск $D^{2} \subset M$, что $D^{2} \cap F=\partial D^{2}$ и кривая $\partial D^{2}$ не ограничивает диска в $F$. Несжимаемая поверхность таких дисков не допускает. Две гомеоморфные поверхности $F_{1}, F_{2} \subset M$ параллельны в $M$, если $F_{1} \cap F_{2}=\partial F_{1}=\partial F_{2}$ и найдется такое отображение $\varphi: F_{1} \times I \rightarrow M$, что $\varphi(x, 0)=x$ для всех $x \in F_{1}, \varphi(x, t)=x$ для всех $x \in \partial F_{1}$ и всех $t \in I, \varphi\left(F_{1} \times\{1\}\right)=F_{2}$ и ограничение отображения $\varphi$ на (Int $\left.F_{1}\right) \times I$ является вложением. Несжимаемый тор $T \subset M$ называется существенным, если он не параллелен граничному тору. Существенное кольцо $A \subset M$ - это собственное несжимаемое кольцо в $M$, не параллельное кольцу в $\partial M$.

ОПРЕДЕЛЕНИЕ. Компактное ориентируемое трехмерное многообразие $B$ называется әлементарным блоком, если выполнено одно из следующих условий:

(1) $B$ является многообразием Зейферта;

(2) $B$ неприводимо, не содержит сушественных торов и колец и не является многообразием Зейферта, причем его край либо пуст, либо состоит из несжимаемых торов.

Блоки этих типов будут называться зейфертовыми и условно гиперболическими, соответственно.

ЗАмечАниЕ. Из доказанной части геометризационной гипотезы Торстона [7] вытекает, что если условно гиперболический блок достаточно велик (т.е. содержит несжимаемую поверхность, в частности, если его край не пуст), то его внутренность обладает полной гиперболической метрикой конечного объема. Вопрос о существовании условно гиперболических, но не гиперболических блоков эквивалентен пока не доказанной части гипотезы Торстона.

ОПРЕДЕЛЕНИЕ. Трехмерное многообразие $M$ назьвается обобщенным графмногообразием, если его можно получить склеиванием элементарных блоков по некоторьм гомеоморфизмам их граничных торов.

Отметим, что граф-многообразия Вальдхаузена [3] по определению составлены из зейфертовых блоков. Таким образом, обобщение состоит в привлечении условно гиперболических блоков. 
ТЕорема 1. Любое компактное ориентируемое неприводимое трехмерное многообразие $M$, край которого либо пуст, либо состоит из несжимаемых торов, является обобщенным граф-многообразием. Более того, многообразие $M$ можно разбить на әлементарные блоки, используя только существенные mopbl.

ДоКАЗАТЕЛЬство. Будем разрезать $M$ по сушественным торам до тех пор, пока это возможно. Процесс заведомо конечен, так как по теореме конечности Хакена [8] любое трехмерное многообразие может содержать только конечное число попарно не параллельных и не пересекающихся несжимаемых поверхностей. В результате мы получим конечный набор неприводимых многообразий, в которых существенных торов уже нет.

Пусть $M_{i}$ - одно из них. Если $M_{i}$ не содержит существенных колец, то оно либо является условно гиперболическим блоком, либо замкнутым многообразием Зейферта без существенных торов. Последнее возможно только когда $M$ расслаивается $\mathrm{c} \leqslant 3$ особыми слоями над сферой или $\mathrm{c} \leqslant 1$ особым слоем над проективной плоскостью. Докажем, что если $M_{i}$ содержит существенное кольцо $A$, то оно является многообразием Зейферта. Обозначим через $L$ ту компоненту связности объединения $A \cup \partial M_{i}$, которая содержит $A$, и через $Y^{3}$ - ее регулярную окрестность в $M_{i}$. Так как граничные окружности кольца $A$ не тривиальны в $\partial M_{i}$, то естественное расслоение кольца $A$ на окружности можно продолжить до расслоения полиэдра $L$ над графом $G$, состолшим из трех ребер и двух вершин валентности 3 . Отсюда следует, что регулярная окрестность $Y^{3}$ также расслаивается на окружности. При этом базой расслоения служит либо диск с двумя дырками, либо лист Мёбиуса с одной дыркой (последнее возможно, когда согласованно ориентированные граничные окружности кольца $A$ лежат на одном и том же граничном торе многообразия $M_{i}$ и имеют там противоположные ориентации).

Так как в $M_{i}$ существенных торов нет, то каждый лежащий внутри многообразия $M_{i}$ граничный тор $T$ многообразия $Y^{3}$ либо параллелен краю, либо сжимаем. Во втором случае тор $T$, как и всякий другой сжимаемый тор в неприводимом многообразии, ограничивает в $M_{i}$ полноторие. При этом меридиан этого полнотория не изотопен слою расслоения, так как в противном случае кольцо $A$ было бы сжимаемым. Отсюда следует, что $M_{i}$ является многообразием Зейферта.

Теорема 1 дает простой способ задания трехмерных многообразий: нужно представить в виде графа схему склеивания элементарных блоков и описать как сами блоки, так и гомеоморфизмы склеивания их граничных торов. К сожалению, каждое многообразие можно разбить на блоки многими различными способами. В этом и следующем параграфе мы покажем, что за счет слияния блоков можно получить минимальное разбиение, которое единственно с точностью до изотопии.

ОпредЕЛЕниЕ. Конечњый набор $T_{1}, \ldots, T_{n}$ попарно непересекающихся несжимаемых торов в трехмерном многообразии $M$ называется минимальной разрезающей системой, если выполнены следующие условия:

(1) торы разрезают $M$ на элементарные блоки;

(2) если какой-нибудь тор $T_{i}$ разделяет два (возможно совпадающих) зейфертовых блока, то для любого выбора зейфертовых расслоений на них индуцированные расслоения на окружности тора $T_{i}$ не изотопны. 
Заметим, что любое компактное ориентируемое неприводимое трехмерное многообразие $M$, край которого либо пуст, либо состоит из несжимаемых торов, содержит минимальную разрезающую систему. Чтобы ее построить, нужно найти некоторое разбиение многообразия $M$ на элементарные блоки с несжимаемьми краями (такое разбиение сушествует по теореме 1). Затем нужно шаг за шагом упрощать его до тех пор, пока это возможно. Каждый шаг состоит в нахождении разрезающего тора $T_{i}$, который нарушает условие $(2)$, и в его удалении. В конце концов мы получим минимальную разрезающую систему.

\section{§3. Грубые и тонкие торы}

ОПреДЕЛЕНИЕ. Существенньй тор $T$ в компактном неприводимом трехмерном многообразии $M$ называется тонким, если найдется такое многообразие Зейферта $Q \subset M$, что

(1) все граничные торы многообразия $Q$ несжимаемы в $M$;

(2) $T$ лежит в $Q$ и существен в нем.

Сушественные торы в $M$, не являюшиеся тонкими, называются грубыми. (Терминология заимствована из гамильтоновой механики (см. [4]).)

ЛЕмма 2. Для любого грубого тора $T_{1} \subset M$ и любого несжимаемого тора $T_{2} \subset M$ найдется такая изотопия $\varphi_{t}: M \rightarrow M, 0 \leqslant t \leqslant 1$, что $\varphi_{1}\left(T_{1}\right) \cap T_{2}=\varnothing$.

ДокАЗАтЕльство. Напомним, что торы $T_{1}, T_{2}$ несжимаемы. Пользуясь приемом устранения самой внутренней окружности, можно уничтожить все тривиальные кривые в $T_{1} \cap T_{2}$. Тогда объединение $T_{1} \cup T_{2}$ расслаивается на окружности, и это расслоение продолжается на регулярную окрестность $N=N\left(T_{1} \cup T_{2}\right)$. Некоторые граничные торы многообразия $N$ могут оказаться сжимаемыми, но тогда они ограничивают в $M$ полнотория вне $N$. Меридианы этих полноторий не изотопны слою многообразия $N$, так как в противном случае слой был бы стягиваем и торы $T_{1}, T_{2}$ были бы сжимаемыми. Поэтому объединение $Q$ многообразия $N$ с полноториями является многообразием Зейферта. По определению грубого тора тор $T_{1}$ обязан быть параллелен краю многообразия $Q$. Поэтому его можно изотопно сдвинуть с $Q$ и, тем самьм, с $T_{2} \subset Q$.

ЗАмечАниЕ. Разумеется, любой конечный набор непересекающихся грубых торов можно изотопно сдвинуть с любого другого набора непересекающихся несжимаемых торов. Более того, изотопию можно выбрать так, чтобы она была неподвижной на тех торах первого набора, которые не пересекают торы второго набора. Доказательство остается в сущности прежним.

ОпрЕДЕлЕНИЕ. Конечный набор попарно не пересекающихся и не параллельных грубых торов в компактном ориентируемом неприводимом многообразии $M$ называется максимальным, если любой грубый тор в $M$, не пересекающий торов набора, параллелен одному из них.

ТЕОремА 3. Для любого компактного ориентируемого неприводимого трехмерного многообразия $M$ максимальная система грубых торов существует и единственна с точностью до изотопии. 
ДокАЗАТЕЛЬСтво. Будем добавлять к пустой системе новые грубые торы до тех пор, пока это возможно, сдвигая каж дый следуюший тор со всех предыдущих по лемме 2. Так как по теореме конечности Хакена [8] любое многообразие может содержать только конечное число попарно не параллельных и не пересекающихся несжимаемых поверхностей, этот процесс заведомо закончится после конечного числа шагов. Поэтому максимальная система сушествует.

Докажем единственность. Пусть $R=\left\{T_{1}, \ldots, T_{k}\right\}$ и $\left\{R^{\prime}=T_{1}^{\prime}, \ldots, T_{m}^{\prime}\right\}-$ две максимальные системы. По лемме 2 мы можем считать, что торы систем не имеют общих точек. Так как система $R$ максимальна, то каждый тор второй системы $R^{\prime}$ параллелен одному из торов системы $R$, причем два тора системы $R^{\prime}$ не могут быть параллельными одному и тому же тору системы $R$. Мы можем заключить, что система $R^{\prime}$ изотопна части системы $R$. Так как система $R^{\prime}$ такжемаксимальна, эта часть совпадает с системой $R$.

ТЕОРема 4. Для любого компактного ориентируемого неприводимого трехмерного многообразия $M$, край которого либо пуст, либо состоит из несжимаемых торов, любая минимальная разрезающая система торов, разбивающая его на әлементарнье блоки, является максимальной системой грубъхх торов.

ДокАЗАТЕЛЬство. Пусть $S=\left\{T_{1}, \ldots, T_{n}\right\}$ - минимальная система разрезающих торов и $R=\left\{T_{1}^{\prime}, \ldots, T_{m}^{\prime}\right\}$ - максимальная система грубых торов. Заметим, что каждый грубый тор $T$ можно изотопно сдвинуть с разрезающих торов, после чего он будет лежать в некотором зейфертовом или условно гиперболическом блоке $B$. В обоих случаях $T$ параллелен одной из компонент края блока $B$ (по определению грубого тора в первом случае и по определению гиперболического блока во втором). Отсюда следует, что $m \leqslant n$ и каждый тор максимальной системы параллелен одному из разрезающих.

Докажем, что любой канонический тор $T=T_{j}$ является грубым. Если $T$ тонок, то он лежит в некотором зейфертовом многообразии $Q \subset M$ с несжимаемым краем, причем сушествен в нем. Обозначим через $Q_{T}$ многообразие Зейферта, получающееся из многообразия $Q$ разрезанием по тору $T$, и через $T_{-}, T_{+}-$копии тора в $\partial Q_{T}$. Так как многообразие $Q_{T}$ не гомеоморфно многообразиям $T^{2} \times I$ или $D^{2} \times S^{1}$, то в $Q_{T}$ найдутся такие послойные существенные кольца $A_{-}, A_{+}$, что $\partial A_{-} \subset T_{-}, \partial A_{+} \subset T_{+}$. Устраним с помощью изотопии колец тривиальные окружности в их пересечении с каноническими торами и несушественные кольца в их пересечении с блоками. Тогда кольца $A_{-}, A_{+}$будут пересекать блоки $B_{-}, B_{+}$, примыкающие к тору $T$, по существенным кольцам. Поэтому ни один из блоков $B_{-}, B_{+}$не может быть условно гиперболическим. Это означает, что $B_{-}, B_{+}$являются многообразиями Зейферта. Напомним, что любое существенное кольцо в многообразии Зейферта послойно (по отношению к некоторой зейфертовой структуре). Поэтому зейфертовы структуры на блоках $B_{-}, B_{+}$можно выбрать так, чтобы их слои на торе $T$ были изотопны. Это противоречит определению минимальной разрезающей системы. Этот же аргумент проходит и в случае $B_{-}=B_{+}$. Тогда край блока состоит из $\geqslant 2$ компонент, и блок содержит существенное кольцо с граничными окружностями в одной из них. Любое такое многообразие Зейферта допускает только одну зейфертову структуру (с точностью до изотопии). 


\section{§4. Простые и специальные спайны}

Напомним, что компактный двумерный полиэдр $P$ называется простылм, если локально он смоделирован на конусе над полным графом с 4 вершинами (см. рис. 1). Это означает, что полиэдру $P$ разрешается иметь только те локальные особенности, которые присутствуют в конусе. Более точно, линк каждой точки полиэдра $P$ должен быть гомеоморфен одному из следующих графов:

(1) окружности;

(2) окружности с диаметром;

(3) окружности с тремя радиусами;

(4) отрезку;

(5) букету трех отрезков с обшим концом.

Объединение точек, имеющих линки типов (4) и (5), представляет собой одномерный полиэдр, который называется краем полиэдра $P$ и обозначается $\partial P$. Компоненты связности множества точек типа (1), т.е. несингулярных точек, называются 2 -компонентами полиэдра $P$. Они являются двумерными многообразиями без края. Точки типа (3) назьваются истинными вершинами полиэдра $P$ (для того, чтобы отличать их, скажем, от вершин клеточных разбиений).

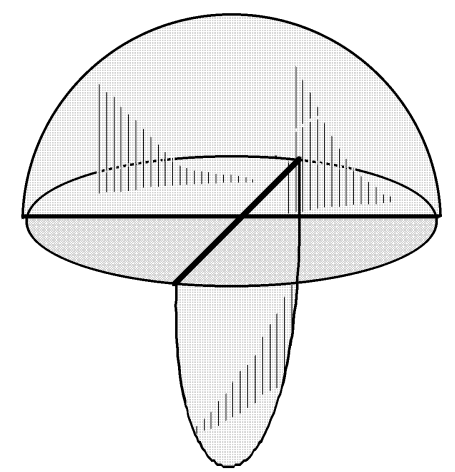

Рис. 1. Конус над полным графом с 4 вершинами

К сожалению, свойство быть простым не является наследственным, так как подполиэдр простого полиэдра не обязан быть простым. Например, он может содержать чисто одномерную часть, состояшую из әлавных ребер, которые не примыкают ни к каким 2-компонентам. Удобно расштирить класс простых полиэдров до минимального класса, который содержит простые полиэдры и обладает свойством наследственности.

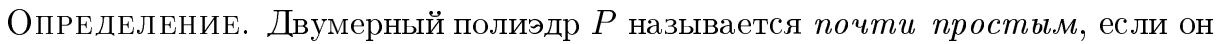
вкладывается в некоторый простой полиэдр.

Легко видеть, что $P$ является почти простым тогда и только тогда, когда линк каждой его точки вкладывается в полный граф с 4 вершинами. Как и выше, точки, линки которых гомеоморфны этому графу, называются истинными вериинами. 
Различие меж ду простыми и почти простыми полиэдрами не очень сушественно. Нетрудно доказать, что каждый почти простой полиэдр можно сколлапсировать либо на граф, либо на набор простых полиэдров, соединенных дугами.

ОПРЕДЕЛЕНИЕ. Простой полиэдр с пустым краем назьвается специальным, если он имеет хотя бы одну вершину и все его 2-компоненты являются 2-клетками.

Простые и специальные полиэдры естественным образом появляются в трехмерной топологии как спайны трехмерных многообразий.

ОПРЕДЕЛЕНИЕ. Подполиэдр $P$ трехмерного многообразия $M$ с непустым краем назьвается его спайном, если разность $M \backslash P$ гомеоморфна $\partial M \times(0,1]$. Под спайном замкнутого многообразия $M$ мы понимаем спайн многообразия $M \backslash \operatorname{Int} D^{3}$, где $D^{3}$ - шар в $M$.

Спайн $P$ называется простылм, почти простым или специальным, если он является полиэдром соответствуюшего типа.

Хорошо известно, что любое компактное трехмерное многообразие имеет простой (и даже специальный) спайн. Более того, по своему специальному спайну трехмерное многообразие восстанавливается однозначно. Для простых спайнов это неверно, так как различные трехмерные многообразия могут иметь гомеоморфные спайны. Тем не менее простые спайны представляют собой удобный способ задания трехмерных многообразий. Разумеется они должны снабжаться дополнительной информацией, достаточной для восстановления соответствующих многообразий.

Пусть $P \subset M$ - простой спайн, представленный в виде клеточного комплекса. Тогда $M$ можно отождествить с тесной регулярной окрестностью спайна $P$. Поэтому клеточная структура спайна $P$ индуцирует разбиение на клетки края многообразия $M$. Имеется естественное отображение $\varphi: \partial M \rightarrow P$, которое переводит клетки в клетки и сохраняет отношение инцидентности. Прообраз при отображении $\varphi$ каждой 2 -клетки в $P$ состоит из двух 2 -клеток. Прообраз ребра может состоять из 1, 2 или 3 ребер, тогда как прообраз вершины может содержать даже 4 точки. Знание клеточной структуры края и индуцированного отображением $\varphi$ соответствия меж ду клетками вполне достаточно для восстановления многообразия $M$.

ЗАМЕЧАНИЕ (О представлении многообразий в памяти компьютера). Любой двумерньй клеточный комплекс может быть представлен в компютере записью информации о числе клеток, об отношении инцидентности между вершинами и ориентированными ребрами и о приклеивающих отображениях 2-клеток. Приклеиваюшие отображения можно задавать строками целых чисел со знаками, которые показывают, как граничные кривые проходят по ориентированным ребрам. Представление трехмерного многообразия $M$ с простым спайном $P$ должно состоять из клеточных представлений спайна $P$ и края $\partial M$ вместе с соответствием между их клетками. Все описанные ниже преобразования многообразий и спайнов могут быть реализованы на этом уровне.

\section{§5. Упрощение простых спайнов}

Упомянутый в $\S 1$ алгоритм распознавания основан на следующем наблюдении (теоретическая подоплека будет приведена в $\S$ 6): структура минимального спайна 
адекватно отражает граф̆-структуру соответствуюшего многообразия. Опишем упрощающие преобразования почти простых спайнов. Они могут быть разбиты на три групाш:

(А) преобразования, которые меняют только клеточную структуру спайна;

(В) преобразования, которые модифицируют спайн, но сохраняют многообразие;

(C) преобразования, которые изменяют и спайн, и многообразие; в этом случае нужно сохранять дополнительную информацию, достаточную для восстановления исходного многообразия по его упрошенной копии.

Пусть $P$ - простой спайн компактного ориентируемого трехмерного многообразия $M$.

ПреОБРАЗОВАниЕ 1 (удаление вершины валентности 2). Пусть $P$ содержит вершину $A$, к которой примыкают ровно два различных ребра $a, b$. Тогда мы объединим эти ребра в одно новое ребро $c$ путем стирания вершины $A$.

ПреоБразование 2 (объединение 2-клеток). Пусть $P$ содержит ребро $a$ валентности 2 , к которому примыкают ровно две различные 2-клетки $X, Y$. Тогда мы объединяем эти клетки в одну новую 2-клетку $Z$ путем стирания ребра $a$.

ПреобРАЗование 3 (удаление иглы). Пусть $P$ содержит ребро $a$ валентности 2, которое имеет свободную вершину. Тогда мы удаляем ребро $a$ вместе с этой свободной вершиной.

Отметим, что преобразования 1-3 относятся к типу (А), т.е. они не меняют ни спайна, ни многообразия (см. рис. 2).

ПреоБРАзовАние 4 (коллапсирование). Пусть 2-клетка $\alpha$ спайна $P$ имеет свободное ребро $a$. Тогда мы удаляем внутренность клетки $\alpha$ вместе с этим свободным ребром.

ЗАмЕчАниЕ. Применяя преобразование 4, нужно быть осторожнњм, так как может получиться почти простой, но не простой спайн. Это случается тогда, когда клетка $\alpha$ имеет другое свободное ребро $b$; после удаления оно становится главньм. Чтобы избежать этого, нужно в таких случаях удалять только часть 2-клетки $\alpha$, оставляя узкую полоску вдоль ребра $b$.

ПРеОБРАЗОВАнИЕ 5 (допустимая переклейка клетки). Пусть $D^{2}$ - такой диск в $M$, что $D^{2} \cap P=\partial D^{2}$ и кривая $\partial D^{2}$ находятся в общем положении в $P$. Тогда диск $D^{2}$ отсекает от пространства $M \backslash P$ шар $B^{3}$. Выберем в простом полиэдре $P \cup D^{2} \subset M$ 2-клетку $\alpha$, отделяющую $M \backslash P$ и $B^{3}$, и удалим ее (см. рис. 3 ). Мы получим новый спайн $P_{1}$ многообразия $M$. Будем говорить, что $P_{1}$ получен из $P$ преобразованием переклейки 2-клетки. Переклейка 2-клетки называется допустимой, если выполнены следующие условия:

(1) это преобразование не увеличивает числа $v(P)$ истинных вершин спайна $P$, т.е. $v\left(P_{1}\right) \leqslant v(P)$;

(2) $v\left(P \cup D^{2}\right)-v(P) \leqslant 4$. 

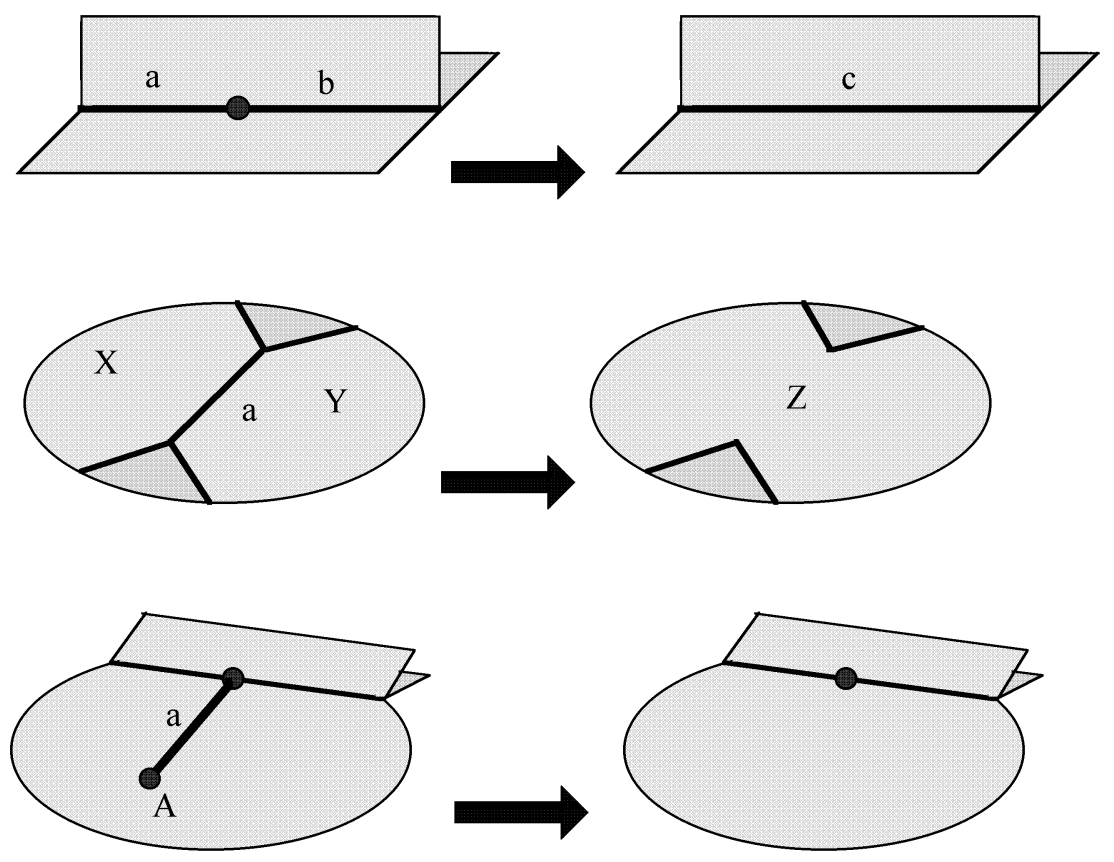

Р ис. 2. Первые три упрошаюших преобразования

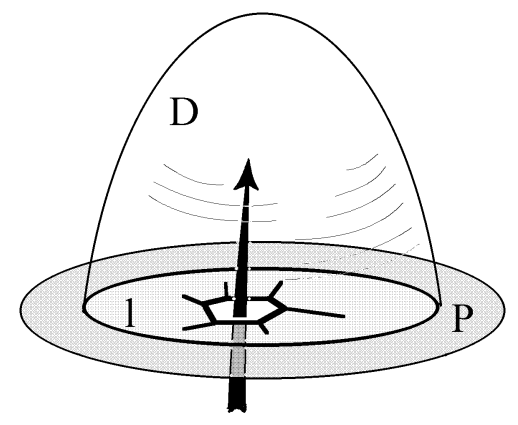

Рис. 3. Переклейка 2-клетки

Преобразование 5 является основным, поэтому мы его прокомментируем более подробно.

(i) Удалять 2-клетку $\alpha$ нужно осторожно, не допуская появления свободных ребер. Как и при преобразовании 4 , нужно оставлять вдоль таких ребер тонкие двумерные полоски.

(ii) Выделим важный частный случай преобразования 5. Пусть 2-компонента $\alpha \subset P$ не является 2-клеткой. Тогда она содержит сохраняюшую ориентацию нетривиальную простую замкнутую кривую $l$, которая может быть 
сдвинута в $\partial M$ двумя различными способами. Допустим, что хотя бы одна из двух полученных окружностей тривиальна в $\partial M$. Тогда $l$ ограничивает такой диск $D^{2} \subset M$, что $D^{2} \cap P=l$, и мы можем упростить $P$ преобразованием 5 .

(iii) Имеется эффективньй способ нахождения всех дисков в $M$, которые удовлетворяют условиям $D^{2} \cap P=\partial D^{2}$ и $v\left(P \cup D^{2}\right)-v(P) \leqslant 4$. Для этого достаточно перечислить все те тривиальные окружности в $\partial M$, которые пересекают прообразы (при отображении $\varphi: \partial M \rightarrow P$ ) тройных ребер спайна $P$ не более чем в 4 точках. Если $l$ - одна из таких окружностей, то кривая $\varphi(l) \subset P$ ограничивает такой диск $D^{2} \subset M$, что $D^{2} \cap P=\partial D^{2}$. Может случиться, что неравенство $v\left(P \cup D^{2}\right)-v(P) \leqslant 4$ не вьполняется из-за лишних вершин, появляющихся в точках самопересечения кривой $\varphi(l)$. Тем не менее, все диски, удовлетворяющие этому неравенству, могут быть построены этим способом.

Заметим, что преобразования 4, 5 имеют тип (В), так как они модифицируют только спайны.

ПреБРАзовАниЕ 6 (разрезание 2-компоненты вдоль нетривиальной окружности). Допустим, что 2-компонента $\alpha \subset P$ содержит нетривиальную сохраняющую ориентацию простую замкнутую кривую $l$. Пусть $A \subset M$ - такое собственное кольцо, что $A \cap \alpha=l$ и пересечение трансверсально. Предположим, что граничные окружности кольца $A$ не тривиальны в $\partial M$ (сравните с замечанием (iii) выше). Удаляя из $P$ открытую регулярную окрестность кривой $l$ в $\alpha$ и удаляя из $M$ открытую регулярную окрестность кольца $A$ в $M$, мы получаем новый простой спайн $P_{1}$ нового многообразия $M_{1}$. Другими словами, $P_{1}$ и $M_{1}$ получаются из спайна $P$ и многообразия $M$ разрезанием вдоль $l$ и $A$, соответственно. Чтобы описать взаимоотношение между многообразиями $M_{1}$ и $M$, обозначим через $L$ ту связную компоненту объединения $A \cup \partial M$, которая содержит $A$. Пусть $Y^{3}$ - регулярная окрестность полиэдра $L$ в $M$. Тогда $M$ можно представить как $M_{1}$ с приклеенным к нему вдоль одного или двух граничных торов многообразием $Y^{3}$. Точно так же, как и в доказательстве теоремы 1 , можно показать, что $Y^{3}$ расслаивается на окружности над диском с двумя дырками или листом Мёбиуса с одной дыркой. Таким образом, преобразование 6 заключается в отщеплении одного зейфертова блока.

ПРеОБРАЗОВАнИЕ 7 (разрезание полосы). Пусть $P$ содержит такую простую дугу $l$, что $l$ лежит в некоторой 2-клетке, а ее концы - на различных свободных ребрах. Преобразование состоит в разрезании полиэдра $P$ по дуге $l$. Чтобы описать соответствующее преобразование многообразия $M$, обозначим через $D^{2}$ такой собственный диск в $M$, что $D^{2} \cap P=l$. Тогда новое многообразие $M_{1}$ получается разрезанием многообразия $M$ по диску $D^{2}$. Другими словами, $M$ можно получить из многообразия $M_{1}$ приклеиванием ручки индекса 1 . Возможны три случая:

(i) если $\partial D^{2}$ не разбивает $\partial M$, то $M=\left(M_{1} \cup D^{3}\right) \#\left(D^{2} \times S^{1}\right)$, где “ $\cup D^{3}$ " означает, что мы заполняем шаром сферическую компоненту края;

(ii) если $D^{2}$ не разбивает многообразия $M$, но $\partial D^{2}$ разбивает $\partial M$, то $M=$ $\left(M_{1} \cup D^{3}\right) \#\left(S^{2} \times S^{1}\right)$

(iii) если многообразие $M_{1}$ состоит из двух компонент связности $M_{1}^{\prime}, M_{1}^{\prime \prime}$, то $M=M_{1}^{\prime} \#\left(M_{1}^{\prime \prime} \cup D^{3}\right)$. 
ПреОБРАЗОвАНИЕ 8 (деликатное прокалывание). Предположим, что $\partial M$ состоит, по крайней мере, из двух компонент, хотя бы одна из которых является сферой. Тогда можно найти такую собственную дугу $l \subset M$, что $l$ пересекает $P$ в одной точке внутри 2-клетки $\alpha$ и соединяет сферическую компоненту края $\partial M \mathrm{c}$ другой компонентой. Удаляя из спайна $P$ клетку $\alpha$ (и оставляя тонкие полоски вдоль могущих появиться главных ребер), мы получаем простой спайн $P_{1}$ такого многообразия $M_{1}$, что $M_{1}=M \cup D^{3}$. Такое прокалывание называется деликатным, поскольку многообразие $M$ меняется совсем незначительно.

ПреоБРАзованиЕ 9 (грубое прокалывание). Мы применяем это преобразование только в случае, когда $\partial M=S^{2}$ и все другие упрощающие преобразования уже исчерпаны. В частности, $P$ должен быть специальньм спайном.

Выберем 2-компоненту $\alpha \subset P$ так, что после ее удаления из спайна $P$ и коллапсирования возникшего полиэдра, мы получим простой полиэдр $P_{1} \subset M$ с наименьшим возможным числом вершин. Тогда $P_{1}$ служит простым спайном своей регулярной окрестности $M_{1}$ в $M$. Ясно, что $M \backslash \operatorname{Int} M_{1}$ является полноторием, т.е. $M$ получается из многообразия $M_{1}$ заполнением Дена.

\section{§6. Алгоритм}

Пусть $M$ - такое компактное ориентируемое трехмерное многообразие, что $\partial M$ либо пуст, либо состоит из торов. Мы хотим разбить $M$ на элементарные блоки, т.е. представить его в виде обобшенного граф-многообразия.

Шаг 1. Построим какой-нибудь простой спайн $P$ многообразия $M$ и представим его в виде клеточного комплекса. Несколько способов построения простых спайнов описаны в [5]. Можно исходить из диаграммы Хегора, представления в виде перестройки сферы по оснашенному зацеплению, триангуляции и т. д.

Шаг 2. Будем применять преобразования 1-9к $P$ до тех пор, пока это возможно. Так как каждое преобразование упрощает спайн, этот процесс закончится после конечного числа преобразований.

Мы получим набор специальных спайнов многообразий, края которых непусты и состоят из торов.

Будем считать эти многообразия нераспознанными (потенциально гиперболическими) блоками, которые нужно проверять на гиперболичность (см. §7).

Шаг 3. Напомним, что каждый раз, когда применяются преобразования типа $(\mathrm{C})$, которые изменяют многообразие, мы запоминаем информацию об этих изменениях. В частности, мы знаем типы зейфертовых блоков, которые были отсечены при преобразовании 6. Мы знаем также, как они склеены друг с другом и как полнотория, которые могли появиться при преобразованиях 7 и 9 , примыкают к ним. Шаг 3 состоит в объединении этих блоков в большие зейфертовы блоки в случаях, когда это возможно (см. §2). В результате мы получим разбиение многообразия $M$ в сумму зейфертовых блоков и нераспознанных кусков, заданных своими специальньпи спайнами.

Напомним, что в теории сложности трехмерных многообразий (см. [5]) сложность компактного трехмерного многообразия $M$ задается формулой $c(M)=\min v(P)$, где $v(P)$ обозначает число истинных вершин спайна $P$, а 
минимум берется по всем почти простым спайнам. Почти простой спайн многообразия $M$ называется минимальныцм, если он имеет в точности $c(M)$ вершин.

ГипотезА 1 (см. [6]). Если специальный спайн $Р$ компактного трехмерного многообразия $M$ не минимален, то число его вериин может быть уменьшено с помощью допустимых переклеек 2-клеток (преобразований типа 6).

ГИПотеЗА 2 (см. [6]). Если специальный спайн замкнутого граф-многообразия минимален, то любое грубое прокальвание (преобразование 9) преобразует его в спайн граф-многообразия.

Справедливость обеих гипотез была проверена с помощью компютера для всех замкнутых многообразий до сложности 8.

ТЕОРема 5. Если минимальный почти простой спайн компактного трехмерного многообразия $M$ специален, то многообразие $M$ неприводимо, имеет несжимаемый (или пустой) край и не содержит существенных колеи.

Доказательство см. в работах [5], [6].

Из теоремы 5 следует, что если гипотеза 1 справедлива, то все нераспознанные блоки, выдаваемые алгоритмом, неприводимы, имеют несжимаемые края и не содержат существенных колец. К сожалению, может случиться так, что некоторые из таких блоков содержат сушественные торы. Простой пример можно получить склеиванием дополнительного пространства зацепления Уайтхеда и некоторого многообразия Зейферта, край которого состоит ровно из одного несжимаемого тора. Тем не менее, при справедливости обеих гипотез алгоритм будет выдавать каноническое разложение для любого граф̆-многообразия Вальдхаузена.

\section{§7. Распознавание гиперболических блоков}

Рассмотрим некоторьй специальный полиэдр $P$. Обозначим через $N(V, P)$ регулярную окрестность в $P$ множества его вершин. Она состоит из нескольких копий конуса над полным графом с 4 вершинами. Конусы над открытыми ребрами графа, т.е. компоненты связности пересечения окрестности $N(V, P)$ с открытыми 2-клетками спайна $P$, будут называться крыльями. Таким образом, $P$ имеет $6 n$ крыльев, где $n$ обозначает число его вершин. Два крыла из окрестности одной вершины называются противоположныцми, если пересечение их замыканий состоит ровно из одной точки (вершины).

Сопоставим каждому крылу некоторое действительное число, называемое его весом, так, чтобы веса противоположных крыльев были равны. Эти веса мы будем рассматривать как неизвестные переменные. Таким образом, имеется всего $3 n$ неизвестных. Эти неизвестные мы подчиним следуюшим условиям совместнос$m u$ :

(1) для каждой вершины полиэдра $P$ сумма весов всех шести прилегающих к ней крыльев должна быть равна $2 \pi$;

(2) для каждой 2-клетки полиэдра $P$ сумма весов всех содержащихся в ней крыльев должна быть равна $2 \pi$.

Условия совместности можно записать в виде системы линейных уравнений. Эта система будет обозначаться через $S(P)$. 
ЗАмечаниЕ. Пусть $P$ является полиэдром некоторого трехмерного $M$ с краем. Тогда он индуцирует двойственную триангуляиию внутренности Int $M$ на 3-симплексы с удаленньми вершинами. Реализуем эти симплексы идеальными тетраэдрами в гиперболическом пространстве $H^{3}$. Если двугранные углы этих тетраэдров взять в качестве весов соответствующих крыльев, то условия совместности первого типа будут выполнены автоматически.

Условия совместности второго типа отвечают требованиям того, что сумма всех двугранных углов вокруг любого ребра двойственной триангуляции должна быть равна $2 \pi$. Таким образом, условия совместности в нашем смысле составляют ровно половину условий совместности В. Торстона (см. [9]). Вместо условий на комплексные параметры идеальных тетраэдров мы накладываем соответствуюшие условия только на их аргументы.

Теорема 6. Пусть $P$ - такой специальный спайн трехмерного многообразия $M$, что система $S(P)$ имеет положительное решение. Тогда $M$ неприводимо, его край $\partial M$ состоит из несжимаемых торов и $M$ не содержит существенных торов и колец.

В силу доказанной части геометризационной гипотезы Торстона теорему 6 можно кратко переформулировать следующим образом: если система $S(P)$ имеет положительное решение, то многообразие $M$ является гиперболическим.

Прежде чем доказывать теорему 6, напомним некоторые понятия теории нормальных поверхностей Хакена [10].

Пусть $\xi$ - разбиение на ручки трехмерного многообразия $M$, состоящее из ручек индекса 0,1 и 2 . Эти ручки называются шарами, балками и плитками, соответственно. Компоненты связности пересечения шаров с балками называются островами, шаров с плитками - мостами. Определим озера как связные компоненты пересечения шаров с краем многообразия $M$.

ОПРеДЕЛЕнИЕ. Собственная поверхность $F \subset M$ называется нормальной (по отношению к разбиению $\xi$ ), если

(1) $F$ пересекает каждую плитку $D^{2} \times I$ по нескольким параллельным листам типа $D^{2} \times\{*\}$;

(2) пересечение поверхности $F$ с каждой балкой $I \times D^{2}$ имеет вид $I \times L$, где $L$ - конечный набор собственных непересекающихся дуг в $D^{2}$, не содержащих замкнутых кривых;

(3) пересечение поверхности $F$ с каждым шаром состоит из дисков (эти диски называются әлементарными);

(4) пересечение края $\partial E$ каждого элементарного диска $E$ с каждьм озером и каждым мостом состоит не более чем из одного отрезка; если озеро и мост прилегают друг к другу, то $\partial E$ может пересекать не более одного из них;

$(5)$ пусть $\partial E$ пересекает озеро $A$ по дуге $l$, тогда концы дуги $l$ должны лежать на различных компонентах связности пересечения озера $A$ с островами.

Будем говорить, что элементарный диск $E$ имеет $m u n(m, n)$, если кривая $\partial E$ пересекает $m$ мостов и $n$ озер. Напомним, что каждый специальный спайн $P$ многообразия $M$ порождает его разбиение на ручки $\xi_{P}$. Чтобы его построить, нужно заменить вершины, ребра и 2-компоненты спайна $P$ на шары, балки и плитки, соответственно. Край каждого шара разбиения $\xi_{P}$ содержит четыре острова, при- 
чем каждые два острова соединены одним мостом. Легко видеть, что элементарные диски для разбиения $\xi_{P}$ могут иметь следующие типы: $(4,0),(3,0),(2,1)$, $(1,2),(0,2),(0,3),(0,4)$. Каждый тип определяет соответствующий диск однозначно (с точностью до гомеоморфизмов шара, переводящих острова в острова и мосты в мосты), кроме типа $(0,3)$, который определяет два элементарных диска $(\mathrm{CM}$ nтx 4$)$

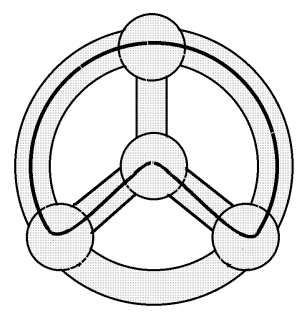

$(4,0)$

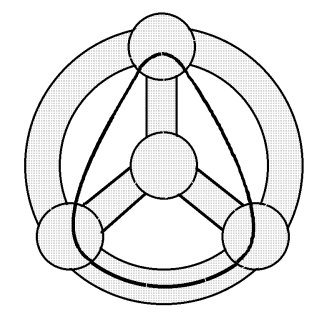

$(1,2)$

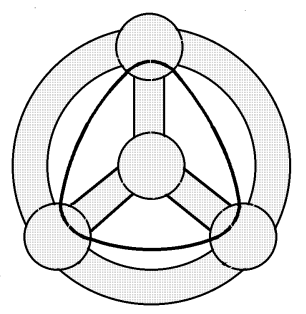

$(0,3)$

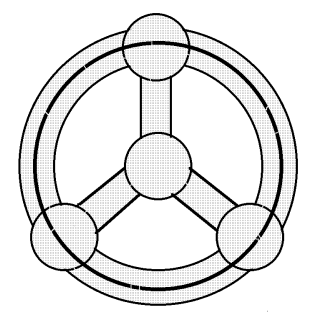

$(3,0)$

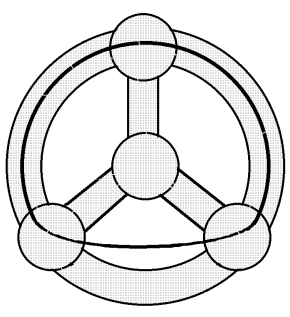

$(2,1)$

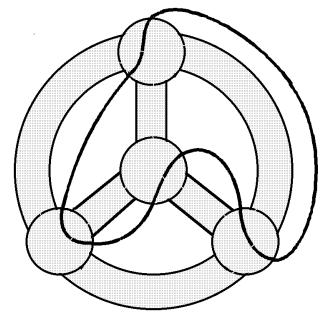

$(0,4)$

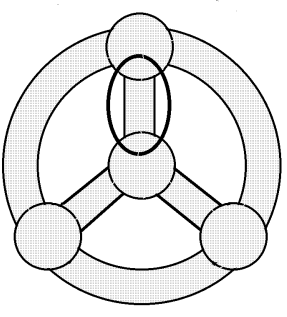

$(0,2)$

Рис. 4. Восемь типов элементарных дисков

Хорошо известно, что любая замкнутая поверхность $F$ в $M$ может быть переведена в нормальную изотопиями, сжатиями и удалениями локальных компонент связности (поверхность локальна, если она содержится в некотором шаре). Каждое сжатие состоит в разрезании тонкой трубочки вдоль края ее меридионального диска и заклеивании двух появляющихся при этом дырок двумя копиями этого диска. Если $F$ имеет край, то дополнительно нужно разрешить сжатия вдоль дисков, опирающихся одной дугой края на $F$, другой - на $\partial M$. 
Допустим, что $M$ содержит одну из следуюших поверхностей:

(1) нетривиальную сферу;

(2) нетривиальный диск;

(3) несжимаемое и не параллельное краю кольцо;

(4) несжимаемый и не параллельный краю тор.

Анализ описанных выше преобразований показьвает, что тогда, по крайней меpe, одна из таких поверхностей может быть найдена среди нормальных.

Поэтому для доказательства теоремы 6 достаточно показать, что многообразие $M$ не содержит нормальных сфер, дисков, колец и торов, кроме торов, параллельных краю.

ДоКАЗАТЕЛЬСТво теОРемЫ 6 . Допустим, что многообразие $M$ содержит такую поверхность $F$, что эйлерова характеристика $\chi(F)$ неотрицательна и $F$ нормальна по отношению к разбиению $\xi_{P}$. Связные компоненты пересечения поверх-

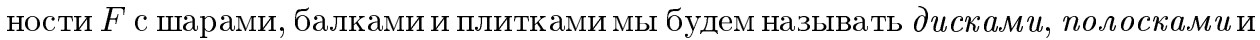
заплатами, соответственно. Эти диски, полоски и заплаты образуют разбиение на ручки $\eta$ поверхности $F$. Компоненты связности пересечения заплат с регулярными окрестностями дисков называются крыльями. Крылья разбиения $\eta$ отвечают крыльям спайна $P$ и потому снабжены положительными весами. Обозначим через $k_{s}$ число дисков разбиения $\eta$, имеюших $s$ приклеенных полосок (с учетом кратности). Так как диск $E$ типа $(m, n)$ имеет в точности $m+n$ приклеенных полосок, $s$ может принимать значения $2,3,4$. Заметим также, что к $E$ примыкают $m$ крыльев и если $m \leqslant 3$, то среди них нет противоположных (по отношению к соответствуюшей вершине спайна $P$ ). Рис. 5 показывает пример разбиения поверхности $F$, обладающей дисками всех 8 возможных типов.

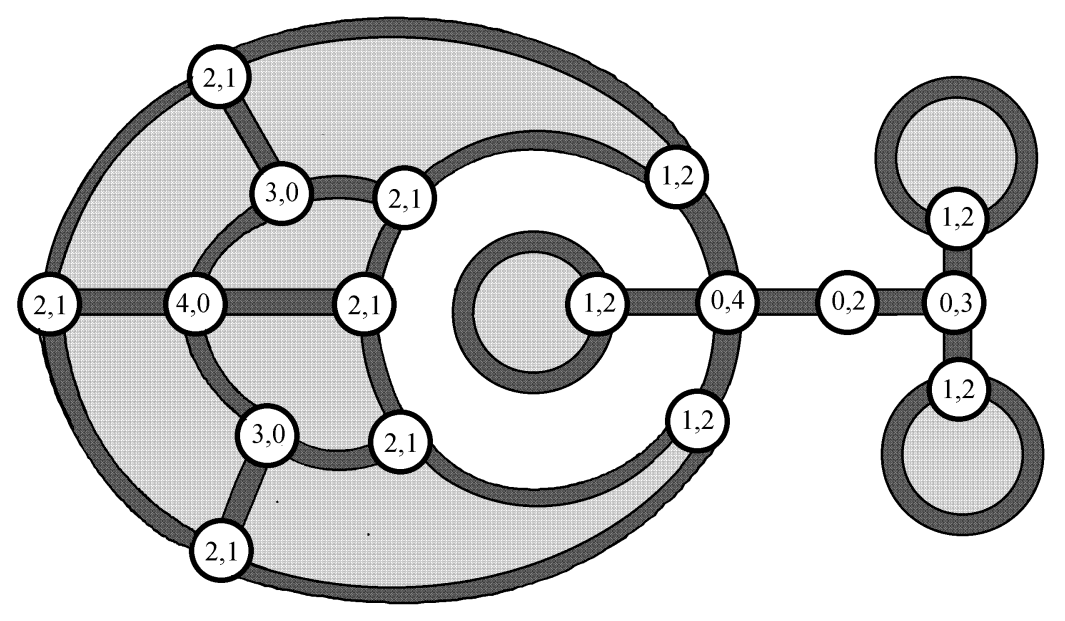

Р ис. 5. Разбиение кольца на диски, полоски и заплаты

Заметим, что $F$ содержит $k_{2}+k_{3}+k_{4}$ дисков и $\left(2 k_{2}+3 k_{3}+4 k_{4}\right) / 2$ полосок. Поэтому $\chi(F)=-k_{3} / 2-k_{4}+p$, где $p$ - число полосок. Так как $\chi(F) \geqslant 0$, мы получаем неравенство

$$
k_{3}+2 k_{4} \leqslant 2 p .
$$


Так как веса крыльев составляют решение системы $S(P)$, общий вес всех крыльев, содержащихся в одной заплате полиэдра $F$, равен $2 \pi$. Поэтому общий вес всех крыльев в $F$ равен $2 \pi p$. Оценим этот вес с помощью рассмотрения крыльев, примькающих к каждому диску $E$. Если $E$ имеет тип $(m, n)$, то к нему примыкает $m$ крыльев. Легко видеть, что их общий вес $w$ может быть оценен следующим обра3ом:

(1) $w<2 \pi$, если $(m, n)=(4,0)$;

(2) $w=\pi$, если $(m, n)=(3,0)$;

(3) $w<\pi$, если $m+n=3$ и $0<m<3$;

(4) $w=0$ во всех остальных случаях, когда $m=0$ и поэтому крыльев нет.

Суммируя по всем дискам, получаем неравенство

$$
\pi k_{3}+2 \pi k_{4} \geqslant 2 \pi p
$$

Более того, равенство возможно, только когда все диски $F$ имеют тип $(3,0)$. Сравнивая неравенства (1) и (2), мы можем заключить, что они в действительности являются равенствами. Это означает, что $\chi(F)=0$ и все элементарные диски в пересечении поверхности $F$ с шарами имеют тип $(3,0)$. Поэтому $F$ является тором, параллельным краю.

\section{Список литературы}

1. Jaco W., Shalen P. Seifert Fibered Spaces in 3-Manifolds // Mem. Amer. Math. Soc. 1979. V. 80.

2. Johannson K. Homotopy Equivalence of 3-Manifolds with Boundaries // Lecture Notes in Math. 1979. V. 761.

3. Waldhausen F. Eine Klasse von 3-dimensionalen Mannigfaltigkeiten I, II // Invent. Math. 1967. V. 3. P. 308-333; // Invent. Math. 1967. V. 4. P. 87-117.

4. Болсинов A.В., Матвеев С.В., Фоменко А. T. Топологическая классификация интегрируемых гамильтоновых систем с двумя степенями свободы. Список систем малой сложности // УМН. 1990. Т. 45. № 2. С. 49-77.

5. Matveev S. V. Complexity Theory of Three-dimensional Manifolds // Acta Appl. Math. 1990. V. 19. № 2. P. 101-130.

6. Matveev S. V. Computer Recognition of Three-Manifolds // Experiment. Math. 1998. V. 7. № 2. P. 153-161.

7. Otal J.-P. Thurston's Hyperbolization of Haken Manifolds // Preprint. Juin 1997, CNRS-UMR 128.

8. Haken W. Some Results on Surfaces in 3-Manifolds // Studies in Modern Topology. MAA Studies in Math. V. 5. Engelwood Cliffs, NJ: Prentice-Hall, 1962. P. 39-98.

9. Thurston W. P. Hyperbolic Geometry and 3-Manifolds // Low-dimensional topology. London Math. Soc. Lecture Note Ser. 1982. V. 48. P. 9-25.

10. Haken $W$. Theorie der Normalfächen. Eine Isotopiekriterium für der Kreisknoten // Acta Math. 1961. V. 105. P. 245-375.

Челябинский государственный университет

E-mail: matveev@csu.ac.ru matveev@cgu.chel.su 